\title{
A Computer Model of Soft Tissue Interaction with a Surgical Aspirator
}

\author{
Vincent Mora, Di Jiang, Rupert Brooks, and Sébastien Delorme \\ Industrial Materials Institute - National Research Council, Canada \\ vincent.mora@imi-cnrc-nrc.gc.ca
}

\begin{abstract}
Surgical aspirators are one of the most frequently used neurosurgical tools. Effective training on a neurosurgery simulator requires a visually and haptically realistic rendering of surgical aspiration. However, there is little published data on mechanical interaction between soft biological tissues and surgical aspirators. In this study an experimental setup for measuring tissue response is described and results on calf brain and a range of phantom materials are presented. Local graphical and haptic models are proposed. They are simple enough for real-time application, and closely match the observed tissue response. Tissue resection (cutting) with suction is simulated using a volume sculpting approach. A simulation of suction is presented as a demonstration of the effectiveness of the approach.
\end{abstract}

\section{Introduction}

Rapidly evolving surgical techniques, patient safety concerns, and the inherent inefficiency of operating room training are strongly driving the need for innovative simulation technologies 1223 . Clinical adoption of virtual reality simulation would result in accelerated training, rapid adoption of new techniques, better surgeries with minimal risk and consequently improved patient care 4. Our research project aims to develop a simulator capable of training medical students to perform surgical resection of brain tumors.

As a fundamental surgical device in neurosurgery [5677, the surgical aspirator must be accurately modelled. This tool has two main functions: (A) aspiration, which is either the non-traumatic removal of blood and fluid or the removal of soft tissue 89, and (B) tissue holding 5]. Surgical aspirators are included in commercial simulators but studies on their mechanical behavior are scarce in the literature and do not provide enough experimental data to develop a model suitable for a simulator [10 11 12 13 14,15 16 17].

A surgical aspirator model is suitable for a simulator if it allows to perform functions $(\mathrm{A})$ and $(\mathrm{B})$ in real time [18. The objective of this study is (1) to measure the interaction between the surgical aspirator and brain tissue, (2) to propose a haptic and graphic model of aspirator tissue interaction based on experimental evidence, and (3) to implement an algorithm for tissue holding and removal with a virtual aspirator. Fluid removal is out of the scope of this paper. As a secondary objective potential phantom material were also experimentally tested in order to find a substitute for brain tissue mechanical testing. 
a)

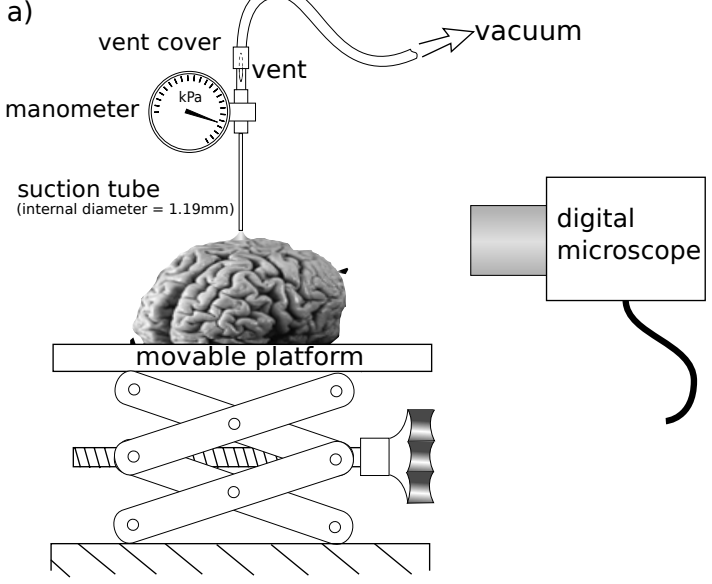

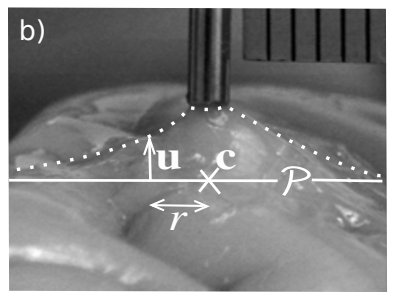

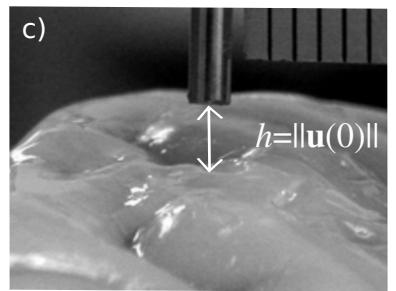

Fig. 1. a) Overview of the experimental setup. b) Photograph taken by the digital microscope before the sample detaches in order to determine the local deformation (white dotted line). Note the ruler used for calibration on the upper right corner. c) Photograph taken by the digital microscope just after the sample detached from the suction tube.

\section{Experimentation}

One calf brain was collected at a slaughterhouse, transported in PBS (phosphate buffered saline) and frozen at $-80^{\circ} \mathrm{C}$. It was then thawed $24 \mathrm{~h}$ prior to the experiment. The tests were carried out on the surface of the calf brain covered by the pia matter with and without moisturizing. The pia matter was removed and the test with moisturizing was repeated. Two phantom materials have been tested: dessert gelatine diluted in boiling water with mass concentration of $5 \%$, $10 \%$ and $15 \%$; and two different brands of soft tofu.

The experimental setup, shown in figure $1 \mathrm{a}$, consisted of a movable platform, a digital microscope and a $1.19 \mathrm{~mm}$ diameter suction tube connected to a vacuum pump. The vacuum hose featured a vent with a sliding cover to vary the suction pressure and a manometer capable of recording negative pressures between $4 \mathrm{kPa}$ and $80 \mathrm{kpa}$ with a $1 \mathrm{kPa}$ precision at the tip of the tube. A ruler was used for photogrammetric calibration, as shown on the upper right corner of figure $1 \mathrm{~b}$ and $\mathrm{c}$.

The experiment proceeded as follows:

1. The material sample was put on the movable platform (see figure 1 a).

2. The vent was totally opened.

3. The sample was raised until it contacted the suction tube. The pressure was recorded when the sample completely blocked the tube oppening.

4. The platform was lowered and photographs were taken at $1 \mathrm{~mm}$ intervals in order to record the shape of the surface in the vicinity of the suction tube. 


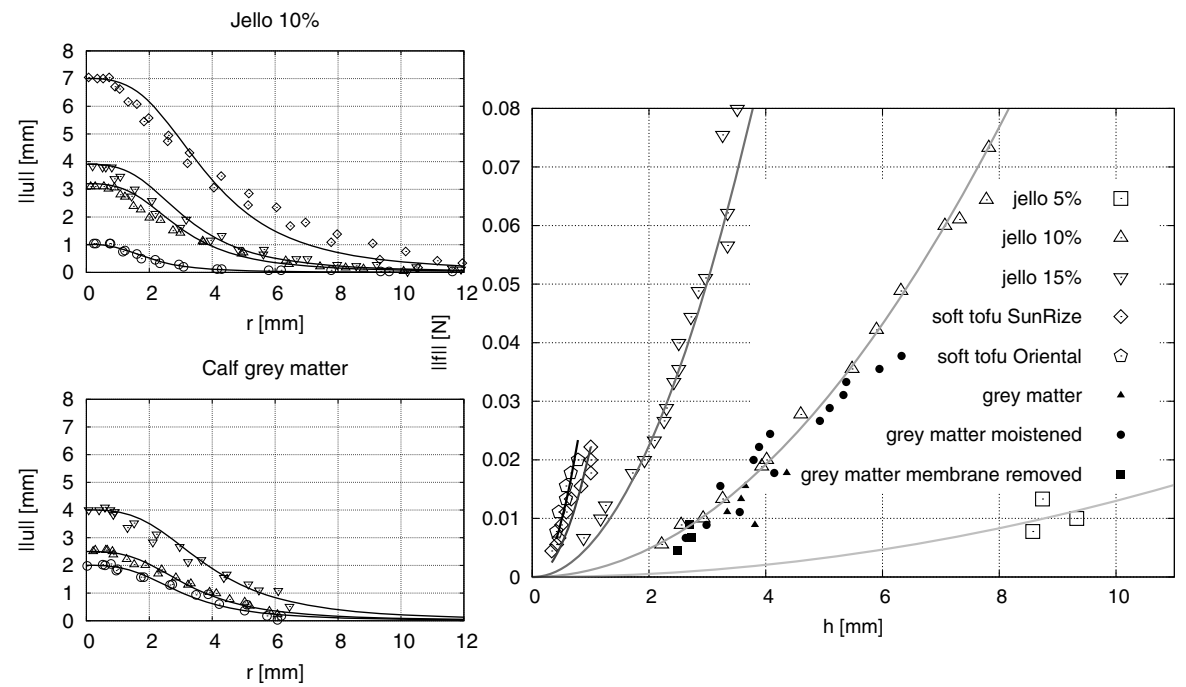

Fig. 2. Left: profile for different value of $h$ along with fits with the function $\|\boldsymbol{u}(r)\|=$ $h^{2} /\left(h+\alpha r^{3}\right)$ (see eq. 2) with $\alpha_{\text {grey matter }}=0.07$ and $\alpha_{\text {gelatine }} 10 \%=0.12$. Right: Force as a function of the vertical displacement $h$ for different materials. The solid lines are fits with the quadratic function $\beta h^{2}$ (see eq. 1) with $\beta_{\text {gelatine } 5 \%}=1.3 \times 10^{-4}$, $\beta_{\text {gelatine } 10 \%}=\beta_{\text {grey matter }}=1.2 \times 10^{-3}, \beta_{\text {gelatine }} 15 \%=5.6 \times 10^{-3}, \beta_{\text {tofu A }}=2.2 \times 10^{-2}$ and $\beta_{\text {tofu } \mathrm{B}}=3.8 \times 10^{-2}$.

5. When the sample detached from the suction tube:

(a) If the surface of the sample was undamaged, a digital photograph was taken. The vent was then further obstructed to increase the negative pressure at the tool tip, and the process went back to step 3 .

(b) If the surface of the sample was damaged, the rupture pressure was recorded and the experiment ended.

Profiles obtained for gelatine $10 \%$ and moisturized calf grey matter without protective membrane are shown in figure 2 The distance $h$ between the surface of the sample and the extremity of the suction tube was measured on the photographs taken just after the sample detached from the tube (see figure1 c). Knowing the internal diameter of the suction tube $d$, the pressure $p$, and assuming frictionless contact between the tube and the sample, the magnitude of the force $f$ when the sample detaches from the tube is given by $\|\boldsymbol{f}\|=\frac{1}{4} \pi d^{2} p$. The experimental data obtained for various materials are shown in figure 2. It can be observed that the calf grey matter has the same behavior as the gelatine $10 \%$ solution with a lower rupture force $(38 \mathrm{mN} \equiv 34 \mathrm{kPa})$ that is further lowered by absence of moisturizing $(18 \mathrm{mN} \equiv 16 \mathrm{kPa})$ or the removal of the protective membrane $(9 \mathrm{mN} \equiv 8 \mathrm{kPa})$. 


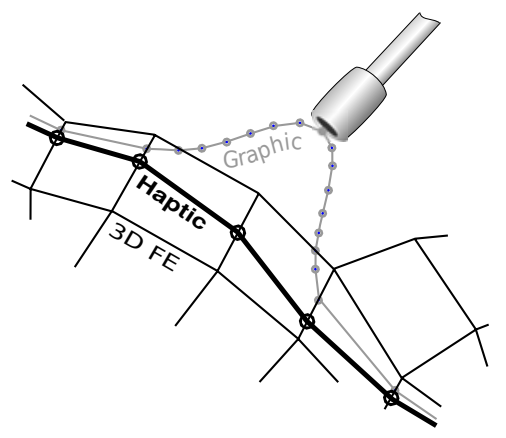

Fig. 3. The three different meshes used in the simulation

\section{Simulating a Surgical Aspirator}

The deformation caused by suction on tissue is both localized and large. It has a typical shape which is important to render to achieve realism in the simulation. Since the phenomenon is localised, a mixed model has been chosen. The global displacement and force fields are given by a 3D explicit non-linear finite element (FE) mode 1 the details of which are outside the scope of this article (see e.g. 19 20] for representative examples). The models for the local displacement and force fields are discussed below.

Tissue deformation can be simulated using FE models. However a FE model would run too slowly to be used for haptics or detailed graphic rendering. Therefore the use of three different models and corresponding meshes is proposed, based on experimental results presented in section 2 ,

1. A 3D FE mesh is used for the deformation calculation.

2. A surface mesh which is a tesselation of an isosurface of a signed distance field defined on the FE mesh, is used for collision detection and haptics rendering.

3. A locally refined copy of the surface mesh is used for graphics rendering.

These three meshes are illustrated in figure 3. A overview of the complete suction simulation is presented in algorithm 1 ,

\subsection{Tissue Holding}

At lower pressures, when close enough to the tissue, tissue is attracted and sticks to the tip of the surgical aspirator. This exerts a force on the tissue, and may be used to hold, or manipulate it. The force $f$ sent to the haptic device, when at distance $h$ from local plane $\mathcal{P}$ (see figure 1), can be expressed as:

$$
\boldsymbol{f}(h)=-\beta h^{2} \boldsymbol{n}
$$

\footnotetext{
${ }^{1}$ Modelling the local surgical aspirator effect directly using a 3D FE model would require a mesh of extraordinary detail, which would not run in real time.
} 


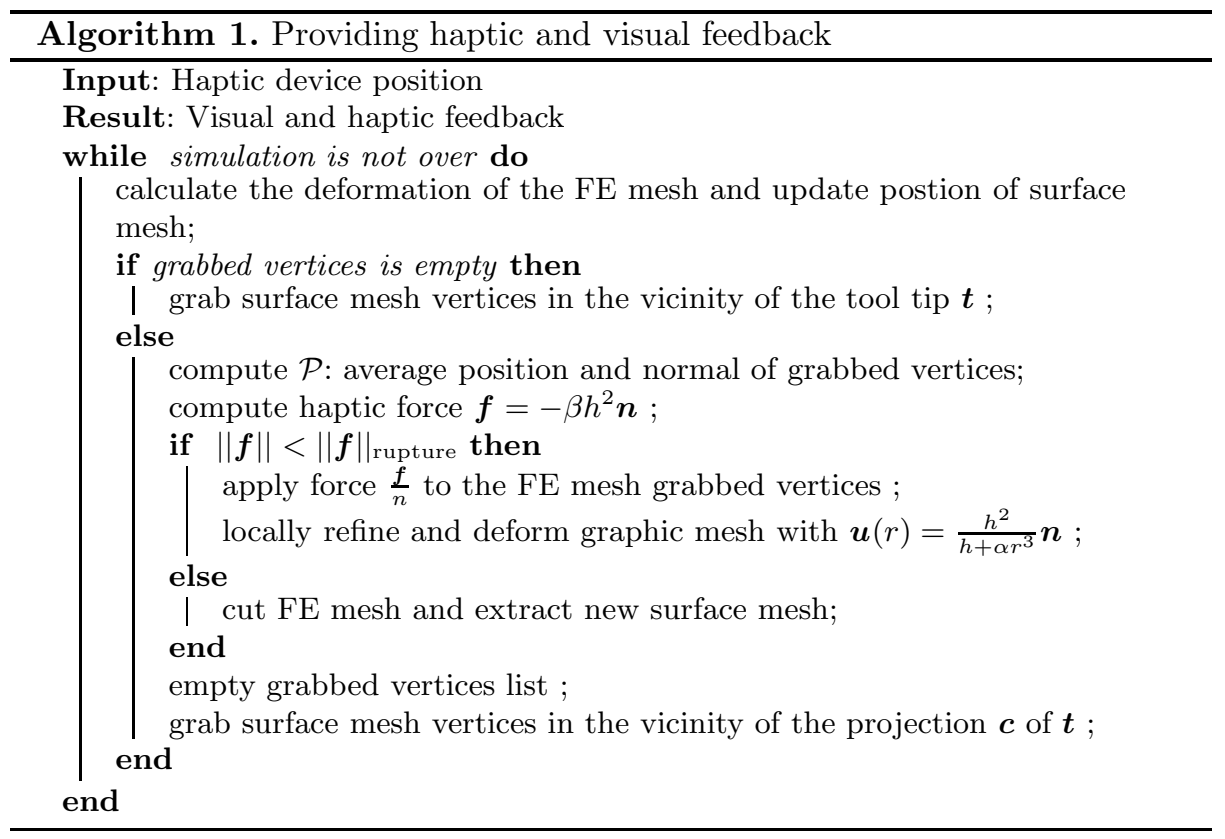

where $\beta$ is a material parameter that increases with the stiffness. This model shows good agreement with experimental data in figure 2. In addition to being rendered by the haptic device, the force can be applied to the $3 \mathrm{D}$ FE model to calculate a deformation. When $\|\boldsymbol{f}\|$ is smaller than the experimentally measured value $\|\boldsymbol{f}\|_{\text {rupture }}$, tissue will be held by the aspirator. Beyond this critical value, the tissue breaks, and is removed by the aspirator as described in the next section.

\subsection{Tissue Removal}

To enable modelling of tissue cutting while avoiding large changes to the $3 \mathrm{D} F E$ mesh a volume sculpting approach [21] is used. The boundaries of soft tissues are modelled as the zero isosurface of a distance field, $F(\boldsymbol{x})$, defined on the nodes of the $3 \mathrm{D}$ FE mesh. Tissue removal is modelled by changing the value of $F$ based on the position of the surgical aspirator. A similar approach has been used to simulate cutting of the petrous bone in 22.

If the aspiration force is greater or equal than $\|\boldsymbol{f}\|_{\text {rupture, a spherical cut- }}$ ting region around the tool tip, $\boldsymbol{t}$, becomes active. Tissue within this sphere is removed. To do this, the distance field is updated according to:

$$
F_{\text {new }}(\boldsymbol{x})=\min \left(F_{\text {old }}(\boldsymbol{x}),\|\boldsymbol{x}-\boldsymbol{t}\|-R\right)
$$

where $R$ is the radius of the cutting sphere.

Once this function is changed, it is necessary to tesselate the new zero isosurface. This is done using one of a family of algorithms, which we refer to as 

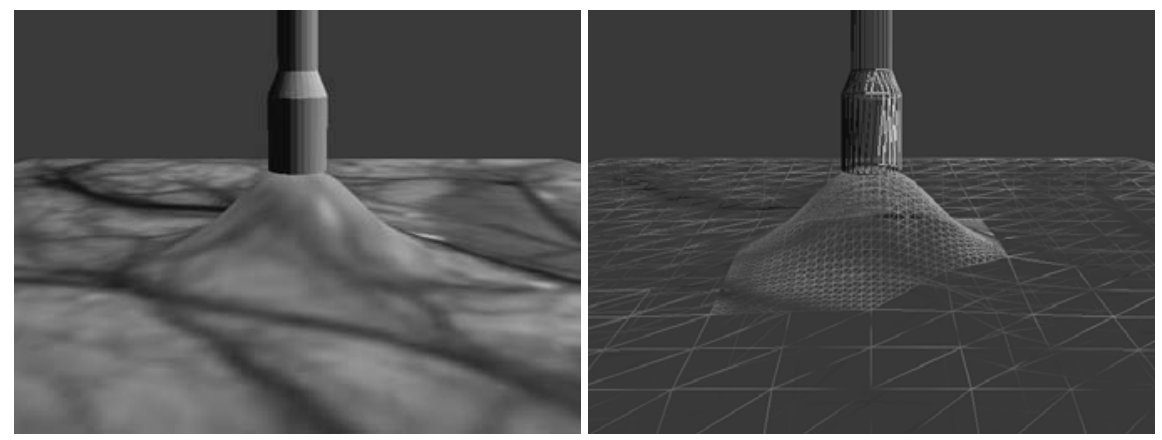

Fig. 4. Simulation of suction effect on tissue using a local rendering model. Left: screenshot from simulation; Right: same showing refined mesh near the surgical aspirator.

marching shapes 23], which tesselate each element given the field values at their vertices. The generated surface consist of triangles, whose vertices lie on the edges of the FE mesh. The most widely known such algorithm is the marching cubes of Lorensen and Cline 24, but the same approach can be used on other shapes, such as tetrahedra, octahedra, etc.

\subsection{Graphics Rendering}

The displacement of a point $\boldsymbol{x}$ on the brain surface due to suction is modelled by the function:

$$
\boldsymbol{u}(r)=\frac{h^{2}}{h+\alpha r^{3}} \boldsymbol{n}
$$

where $\alpha$ is a material parameter, $h$ is the distance between the tool tip $t$ and the local plane $\mathcal{P}, r$ is the distance between $\boldsymbol{x}$ and the projection $\boldsymbol{c}$ of $\boldsymbol{t}$ on $\mathcal{P}$, and $\boldsymbol{n}$ is the normal to $\mathcal{P}$ (see figure $1 \mathrm{~b}$ ). Figure 2 shows good agreement of the model with the experimental data.

The triangle elements of the surface mesh that are close to the suction tool are recursively subdivided into smaller triangles. All the nodes of the mesh are then displaced according to the model. Figure 4 shows a screenshot of the simulated interaction between surgical aspirator and brain tissue with a surface mesh that is locally refined for graphics rendering. When cutting is taking place, the use of this graphic model gives the appearance that the interior parts of the tissue pops up into the aspiration tool.

\section{Discussion}

In this paper, we have described a model to simulate the effect of a surgical aspirator on tissue. By separating the haptic and graphical models from the FE model, the experimentally observed haptic and visual effects of aspirating tissue were simulated in a computationally efficient manner. 
a)

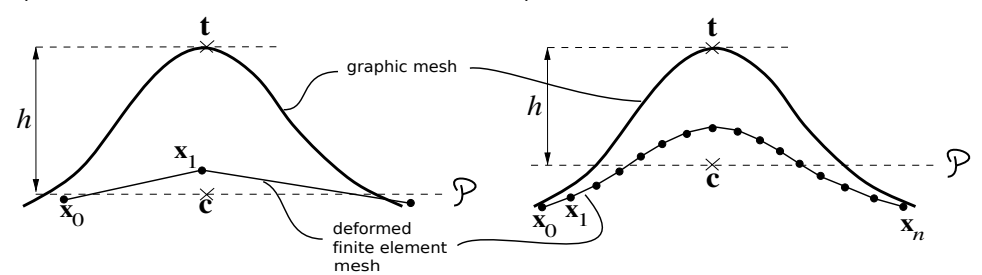

Fig. 5. Definition of $\mathcal{P}$ and $h$ when the $3 \mathrm{D}$ FE mesh is a) coarse, b) fine

The experiments with phantom material suggest that gelatine $(10 \%$ mass concentration) could be an adequate substitute for brain for mechanical testing purposes. This preliminary result must be validated using fresh brain tissue since freezing can alter the properties of biological tissues [25]. Other work [26] has shown that these effects can be compensated by applying a corrective factor. Thus we think it likely that with further work a suitable concentration of gelatine can be found to mimic the properties of fresh brain.

The proposed model is limited by the dependency of the definition of the local plane $\mathcal{P}$ on the refinement of the 3D FE mesh (see figure 5). In the experiment $\mathcal{P}$ (figure 1) is the average plane of the undeformed brain surface. In the model $\mathcal{P}$ (algorithm 1) is the average plane of the surface of the deformed 3D FE mesh. If the $3 \mathrm{D} \mathrm{FE}$ mesh is coarse, compared to the local deformation caused by the aspirator, the local deformation of the $3 \mathrm{D} \mathrm{FE}$ mesh is small and the model $\mathcal{P}$ is close to the experimental $\mathcal{P}$. However if the $3 \mathrm{D} \mathrm{FE}$ mesh is fine, its local deformation will cause the model $\mathcal{P}$ to diverge from the experimental $\mathcal{P}$ which affects the calculation of $\boldsymbol{f}(h)$. This problem is not a real concern in our case since if the finite element mesh can be made fine enough for this problem to be significant, the mesh would then be fine enough to avoid the need for this local model altogether.

Future work will focus on modelling the interaction of other neurosurgical tools such as the Cavitron Ultrasonic Surgical Aspirator (CUSA), as well as on validating the proposed model against a $\mathrm{FE}$ simulation results obtained with a fine mesh, and against experimental data obtained on fresh human brain tissue.

\section{References}

1. Vuskovic, V., Kauer, M., Szekely, G., Reidy, M.: Realistic force feedback for virtual reality based diagnostic surgery simulators. In: Proceedings of the ICRA (2000)

2. Spicer, M.A., Apuzzo, M.L.: Virtual reality surgery: neurosurgery and the contemporary landscape. Neurosurgery 52(3), 489-498 (2003)

3. Rosenthal, R., et al.: The future of patient safety: surgical trainees accept virtual reality as a new training tool. Patient Safety in Surgery 2 (2008)

4. Mauro, A.D., Raczkowsky, J., Wirtz, R., Wörn, H.: Development of a microscope embedded training system for neurosurgery. Biomedical Simulation, 209-214 (2008)

5. Yasargil, M.G.: Microneurosurgery: microsurgery of CNS tumors. Thieme (1996)

6. Cuatico, W.: Neurosurgical suction tips. Acta Neurochirurgica 46, 303-306 (1979) 
7. Vällfors, B.: Neurosurgical suction systems, an experimental study. Thesis, Göteborg, Sweden (1976)

8. Vällfors, B.: Efficient atraumatic liquid suction by means of slit suction tubes combined with a pressure control unit. Neurosurgical Review 7, 179-183 (1984)

9. Maxwell, R.E., Chou, S.N.: Convexity meningiomas and general principles of meningioma surgery. In: Operative Neurosurgical Techniques: Indications and Methods, pp. 491-501. Grune and Stratton, New York (1982)

10. Papadopoulos, S.M., McGillicuddy, J.E.: A simple device for controlling suction force in neurosurgical operations. Neurosurgery 25, 662-663 (1989)

11. Johnson, J.P., Becker, D.P.: A continuous microneurosurgical irrigation and suction system: technical note. Neurosurgery 39, 409-411 (1996)

12. Shalit, M.N.: A simple method for the control of suction power during neurosurgery. Acta Neurochirurgica 64, 235-236 (1982)

13. Lefranc, F., Brotchi, J.: Performance of a new type of suction tip attachment during intramedullary tumor dissection: technical note. Neurosurgery 61 (2007)

14. Aoki, T., Ohashi, T., Matsumoto, T., Sato, M.: The pipette aspiration applied to the local stiffness measurement of soft tissues. Annals of Biomedical Engineering 25, 581-587 (1997)

15. Vällfors, B.: Suction in neurosurgery. Acta Neurochirurgica 55, 35-42 (1980)

16. Mazza, E., Grau, P., Hollenstein, M., Bajka, M.: Constitutive modeling of human liver based on in vivo measurements. In: Metaxas, D., Axel, L., Fichtinger, G., Székely, G. (eds.) MICCAI 2008, Part II. LNCS, vol. 5242, pp. 726-733. Springer, Heidelberg (2008)

17. Schiavone, P., Boudou, T., Promayon, E., Perrier, P., Payan, Y.: A light sterilizable pipette device for the in vivo estimation of human soft tissues constitutive laws. In: Proceedings of the 30th EMBC, pp. 4298-4301 (2008)

18. Berkley, J., Weghorst, S., Gladstone, H., Raugi, G., Berg, D., Ganter, M.: Fast finite element modeling for surgical simulation. In: Medicine Meets Virtual Reality, pp. 55-61. IOS Press, Amsterdam (1999)

19. Taylor, Z.A., Cheng, M., Ourselin, S.: High-speed nonlinear finite element analysis for surgical simulation using graphics processing units. Transactions on Medical Imaging 27(5), 650-662 (2008)

20. Miller, K., Joldes, G., Lance, D., Wittek, A.: Total lagrangian explicit dynamics finite element algorithm for computing soft tissue deformation. Communications in Numerical Methods in Engineering 23, 121-134 (2007)

21. Galyean, T.A., Hughes, J.F.: Sculpting: an interactive volumetric modeling technique. Computer Graphics 25(4), 267-274 (1991)

22. Pflesser, B., Petersik, A., Tiede, U., Hohne, K.H., Leuwer, R.: Volume cutting for virtual petrous bone surgery. Computer Aided Surgery 7(2), 74-83 (2002)

23. Newman, T.S., Yi, H.: A survey of the marching cubes algorithm. Computers and Graphics 30, 854-879 (2006)

24. Lorensen, W., Cline, H.: Marching cubes: a high resolution 3d surface construction algorithm. Computer Graphics 21(4), 163-169 (1987)

25. Fallenstein, G.T., Hulce, V.D., Melvin, J.W.: Dynamic mechanical properties of human brain tissue. Journal of Biomechanics 2(3), 217-226 (1969)

26. Lippert, S., Grimm, M.: Estimating the material properties of brain tissue at impact frequencies: A curve-fitting solution. In: Summer Bioengineering Conference, Key Biscayne, Florida (2003) 